\title{
Platinum Doublet
}

National Cancer Institute

\section{Source}

National Cancer Institute. Platinum Doublet. NCI Thesaurus. Code C162261.

A drug combination of a platinum agent, such as cisplatin (CDDP), carboplatin (CBDCA) or nedaplatin (CDGP), and a third-generation agent, such as docetaxel (DTX), paclitaxel (PTX), vinorelbine (VNR), gemcitabine (GEM), irinotecan (CPT-11), pemetrexed (PEM), or tegafur/gimeracil/oteracil (S-1). 